\title{
Research Paper: Paeonol Protection Against Intrastriatal 6-Hydroxydopamine Rat Model of Parkinson's Disease
}

\author{
Jamileh Ghalami $^{1}$ (i), Tourandokht Baluchnejad Mojarad ${ }^{*}$ (D), Monireh Mansouri ${ }^{1}$ (i), Safoura Khamse ${ }^{1}$ (i), Mehrdad Roghani ${ }^{2}$ (i)
}

1. Department of Physiology, School of Medicine, Tehran University of Medical Sciences, Tehran, Iran.

2. Neurophysiology Research Center, Shahed University, Tehran, Iran.

\begin{tabular}{|l|l|l}
\hline $\begin{array}{l}\text { Use yur devic to scan } \\
\text { and read thearticle online }\end{array}$ & $\begin{array}{l}\text { Crtation: Ghalami, J., Baluchnejad Mojarad, T., Mansouri, M., Khamse, S., \& Roghani, M. Paeonol Protection Against In- } \\
\text { trastriatal 6-Hydroxydopamine Rat Model of Parkinson's Disease. Basic and Clinical Neuroscience, 12(1), 43-56. http://dx.doi. } \\
\text { org/10.32598/bcn.12.6.88.7 }\end{array}$ \\
dolihttp://dx.doi.org/10.32598/bcn.12.6.88.7
\end{tabular}

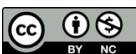

Article info:

Received: 08 Jun 2018

First Revision: 24 Jul 2018

Accepted: $24 \mathrm{Jul} 2019$

Available Online: 01 Jan 2021

Keywords:

Paeonol, 6-hydroxydopamine, Oxidative stress, Apoptosis, GABA receptor, Metabotropic glutamate receptor

\begin{abstract}
A B S T RA C T
Introduction: Parkinson's Disease (PD) presentations comprise frequent movement disorders in the elderly with various symptoms consisting of motor and non-motor complications. Paeonol is a phenolic chemical agent that has shown antioxidant and anti-inflammatory effects in different disorders and promising effects on metabotropic glutamate receptors (mGluR)- and GABAA-mediated neurotransmission. In this research, we tried to show the neuroprotective potential of paeonol in rat PD model induced by intrastriatal 6-hydroxydopamine (6-OHDA).

Methods: Rats with intrastriatal 6-OHDA lesioning received with paeonol at a dosage of $100 \mathrm{mg} / \mathrm{kg} / \mathrm{d}$ for one week. In the end, some biomarkers of oxidative stress, apoptosis, and astrogliosis in nigral and striatal tissues were evaluated in addition to behavioral and Tyrosine Hydroxylase (TH) immunohistochemical analysis.

Results: The obtained data showed that paeonol alleviates apomorphine-induced rotations and reduces the delay time to initiate and the total time in the narrow beam test. However, its beneficial behavioral effect vanished after intracerebroventricular administration of mGluR III or GABAA receptor antagonists. Moreover, paeonol significantly restored striatal malondialdehyde, tissue levels of reactive oxygen species, the activity of the protective and vital enzymes consisting of superoxide dismutase and catalase, Glial Fibrillary Acidic Protein (GFAP), DNA fragmentation, phosphor apoptosis signal-regulating kinase 1, and nigral aquaporin 4 with no significant and proper change of nitrite, interleukin- $1 \beta$, inducible nitric oxide synthase, and angiotensin II. Additionally, paeonol prevented injury and reduced tyrosine hydroxylase-containing neurons in the midbrain nigral tissue.
\end{abstract}

Conclusion: These obtained findings evidently designate neuroprotective property of paeonol in 6-OHDA murine model of PD that is exerted via easing of oxidative stress, apoptosis, astrogliosis, and its advantageous effect is to some extent mediated via mGluR III/GABAA pathway.

\section{* Corresponding Author:}

Tourandokht Baluchnejad Mojarad, PhD.

Address: Department of Physiology, School of Medicine, Tehran University of Medical Sciences, Tehran, Iran.

Tel: +98 (912) 4549337

E-mail:tmojarad@yahoo.com 


\section{Highlights}

- Paeonol alleviated motor abnormalities.

- Administration of mGluR III or GABAA receptor antagonists ameliorated beneficial effect of paeonol.

- Paeonol reduced oxidative stress, astrogliosis and apoptosis.

- Paeonol exerted a protective effect on tyrosine hydroxylase-containing neurons in the nigral tissue.

\section{Plain Language Summary}

Parkinson's Disease is known as a frequent neurological disorder in the elderly. This disorder is associated with various symptoms that are difficult to manage and/or to treat. Paeonol is a natural chemical with antioxidant property. In this study, we tried to show beneficial effect of paeonol in an animal model of Parkinson's disease. Our findings showed that paeonol reduces motor complications, lowers oxidative damage and preserves related neuronal cells against insulted damage. It seems that paeonol may be a promising agent for ancillary management of Parkinson's disease.

\section{Introduction}

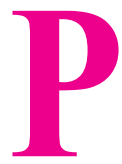

arkinson's Disease (PD) is presented as movement disorders that cause debilitating symptoms in older adults with neurodegenerative and progressive nature. It principally instigates by the demise of midbrain substantia nigra pars compacta (SNC) dopaminergic neurons (Braak, Ghebremedhin, Rub, Bratzke, \& Del Tredici, 2004; Hornykiewicz \& Kish, 1987). PD accompanies cardinal motor symptoms such as resting tremor, rigidity, bradykinesia (or akinesia), and postural imbalance (Dauer \& Przedborski, 2003). Oxidative stress is caused by the excess generation of Reactive Oxygen Species (ROS), the development of imbalance in antioxidant elements (Ahmad et al., 2005; Kiasalari, Khalili, Baluchnejadmojarad, \& Roghani, 2016), and the appearance of inflammation (Gustot et al., 2015; Ren, Tian, Yin, Sun, \& Zhang, 2016; Spittau, 2015). All of these anomalies play principle roles in the development of PD. The Renin-angiotensin system has been recognized as one of the effectors of oxidative stress, and angiotensin II is a major effector of inflammation in the brain, as reviewed before (Prusty, Sahu, \& Subudhi, 2017). The water-selective membrane transport protein aquaporin 4 is also upregulated in astrocytes in inflammation-related disorders such as PD (Sun et al., 2016), and its deficiency causes easier degeneration of dopaminergic neuronal cells in PD (Zhang et al., 2015). Astrogliosis (Sun et al., 2016), apoptosis (Srivastav, Fatima, \& Mondal, 2017), and related Apoptosis Signal-Regulating Kinase (ASK1) (Ouyang \& Shen, 2006; Ray, Sehgal, Karunakaran, Rangarajan, \& Ravindranath, 2015) have also been postulated as key players in the pathogenesis of PD. Despite achievements in the synthesis of pharmacologic products and employment of appropriate surgical methods (i.e. deep brain stimulation), a definite pharmacological solution to postpone disease progression is still missing (Beitz, 2014).

Paeonol (2'-hydroxy-4'-methoxyacetophenone) is a major phenolic and active constituent of Paeonia suffruticosa Andrews (its root bark) and Paeonia lactiflora Pall (its root) with multiple beneficial effects in oriental medicine $(\mathrm{Wu}$, Wu, \& Chen, 2010). Paeonol has shown various pharmacological effects like anti-atherosclerotic (Hu et al., 2012), suppression of oxidative stress (Choy, Lau, Murugan, \& Mustafa, 2017; Ping et al., 2014), inflammation (Liao et al., 2016; Liu, Feng, Wang, Zhao, \& Li, 2017; Lou et al., 2017), anti-diabetic (Lau et al., 2007), antidepressant-like effects (Tao et al., 2016), and protective effect in cerebral ischemic conditions (He, Cai, Li, \& Guo, 2018; Zhao et al., 2018). Besides, paeonol can improve cognition in diabetic rats (Liu et al., 2013) and exert a protective and anti-Alzheimer effect following intra-hippocampal injection of Aß1-42 (Zhou et al., 2011). Recently, paeonol showed therapeutic effects in methyl-4-phenyl-1,2,3,6tetrahydropyridine/probenecid-induced PD model in the mice decreases the damage due to oxidative stress and neuroinflammation and by exerting a neurotrophic effect on dopaminergic neurons (Shi, Chen, Liu, \& Qu, 2016). A portion of the neuroprotective potential of paeonol has been explained by its ability to lower glutamate neurotoxicity and inhibit apoptosis (Wang et al., 2011). In this regard, it has shown that a combination of mGluR III agonist and mGluR I antagonist exerts robust nigrostriatal neuropro- 
tection in the 6-OHDA-developed model of PD (Vernon, Croucher, \& Dexter, 2008). Moreover, it has been claimed that paeonol could target GABAA receptors (Sun, Qiao, \& Zhang, 2011). Of related importance, GABAA activation is essential for some natural compounds to exert a protective effect in hemiparkinsonian rats (Yunes, Casas, Gaglio, \& Cabrera, 2015). This study was conducted to find the other neuroprotective property of paeonol in the 6-OHDAdeveloped murine model of PD and unravel some underlying modes of action.

\section{Materials and Methods}

\subsection{Animals and experimental procedure}

Adult rats (a total of 72 male Wistar strain, a bodyweight of 200-250 g) were purchased from the rodents breeding facility of Iran University of Medical Sciences (IUMS). The animals were kept in the recommended standard situation (12/12 h light/dark periods, lights on at $8 \mathrm{PM}$ ). Used procedures on animals were certified by the local Ethics Committee of Iran University of Medical Sciences in 2015.

Rats were randomly placed into 6 testing groups: 1) sham-operated (sham, $\mathrm{n}=8$ ), 2) paeonol-pretreated sham (sham+paeonol, $\mathrm{n}=8$ ) receiving paeonol with a dosage of $100 \mathrm{mg} / \mathrm{kg}, 3$ ) lesion group (6-OHDA, $\mathrm{n}=8$ ), 4) lesion group under treatment with paeonol at a dosage of $100 \mathrm{mg} / \mathrm{kg}$ (6-OHDA+paeonol, $\mathrm{n}=8)$, 5) lesion group receiving paeonol at a dose of $100 \mathrm{mg} / \mathrm{kg}$ and receiving specific group III metabotropic glutamate (mGluR III) receptor antagonist (6OHDA+paeonol $+\mathrm{MPPG}, \mathrm{n}=8)$, and 6) lesion group under treatment with paeonol at a dosage of $100 \mathrm{mg} / \mathrm{kg}$ plus GABAA receptor antagonist (6-OHDA+paeonol+SR-95531, n=8). For making murine PD model, the specific neurotoxin (6-OHDA) was targeted into the left striatum in deeply anesthetized animals (ketamine at a dosage of $80 \mathrm{mg} / \mathrm{kg}$ and xylazine at a dosage of $8 \mathrm{mg} / \mathrm{kg}$, both intraperitoneally). For the surgical procedure, the animals were placed in a stereotaxic device (Stoelting Co., USA) at the following coordinates: $3 \mathrm{~mm}$ lateral and $0.2 \mathrm{~mm}$ anterior versus bregma and $5 \mathrm{~mm}$ inferior to the dura according to rat stereotaxic atlas (Paxinos \& Watson, 1986). The 6-OHDA lesioned rats were injected with $5 \mu \mathrm{L}$ of normal saline containing $2.5 \mu \mathrm{g} / \mu \mathrm{L}$ of 6-OHDA hydrochloride (Sigma-Aldrich, USA) and $0.2 \%(\mathrm{w} / \mathrm{v})$ ascorbate.

The animals in the sham group had an intrastriatal injection of a normal saline-ascorbate combination. The paeonol-pretreated 6-OHDA groups were treated with 6-OHDA in addition to paeonol (2'-hydroxy-4'- methoxyacetophenone; Sigma-Aldrich, the USA, with a purity greater than $99 \%$ ) solubilized in Kolliphor at a dosage of $100 \mathrm{mg} / \mathrm{kg} / \mathrm{d}$. Paeonol was daily administered per os (PO), started 1 week till one hour before the surgery. The dose of paeonol was derived from earlier research investigations on its beneficial effects in lessening cognitive impairment and neurotoxicity induced by dgalactose in ICR mice (Zhong, Ge, Qu, Li, \& Ma, 2009) and its hypoglycemic and antioxidant activity in a streptozotocin-produced model of diabetic encephalopathy in the rat (Liu et al., 2013). Two lesion groups received paeonol in addition to 6-OHDA, and $2 \mu \mathrm{L}$ of $2.5 \mu \mathrm{g} / \mu \mathrm{L}$ of selective III metabotropic glutamate (mGluR III) receptor antagonist Magnesium Pyridoxal 5-phosphate Glutamate (MPPG) ((RS))- $\alpha$-methyl-4-phosphonophenylglycine dissolved in dimethyl sulfoxide with further dilution in artificial Cerebrospinal Fluid (aCSF); SigmaAldrich, USA) or $2 \mu \mathrm{L}$ of $0.5 \mu \mathrm{g} / \mu \mathrm{L}$ of specific GABAA receptor antagonist SR-95531 (gabazine or 2-(3-carboxypropyl)3amino-6-(4 methoxyphenyl) pyridazinium bromide dissolved in aCSF; Sigma-Aldrich, the USA) $30 \mathrm{~min}-$ utes after the final administration of paeonol. The used aCSF solution was made of $20 \mathrm{mM}$ of $\mathrm{NaCl}, 1.15 \mathrm{mM}$ of $\mathrm{CaCl} 2,3 \mathrm{mM}$ of $\mathrm{KCl}, 0.8 \mathrm{mM}$ of $\mathrm{MgCl} 2,27 \mathrm{mM}$ of $\mathrm{NaHCO} 3$, and $0.33 \mathrm{mM}$ of $\mathrm{NaH} 2 \mathrm{PO} 4$ with $\mathrm{pH}$ at 7.2). The antagonists were ICV given at the following coordinates: $1.4 \mathrm{~mm}$ lateral and $1 \mathrm{~mm}$ posterior versus bregma, $3.6 \mathrm{~mm}$ inferior to the dura. The pure effect of these antagonists was examined in an earlier pilot study in 6-OHDA lesioned rats in the absence of paeonol. In this respect, no statistically significant modification was noted versus 6-OHDA lesioned group with regard to our used behavioral parameters.

\subsection{Behavioral evaluation}

After one week, the behavioral tests were done by an experienced observer who was blind to treatments. These tests were done between $10 \mathrm{AM}$ and $3 \mathrm{PM}$ in a semi-dark and quiet room.

\subsubsection{Elevated narrow beam task}

The procedure for this test has been reported (Kiasalari, Baluchnejadmojarad, \& Roghani, 2016). The used device had a wooden beam (width: $4 \mathrm{~cm}$, length: $105 \mathrm{~cm}$, height: $3 \mathrm{~cm}$ ) at the height of $80 \mathrm{~cm}$. Starting mark was $20 \mathrm{~cm}$ distant from its end. During the test, the rat was placed on the starting area, and after its release, time was taken to reach the starting mark. This time denotes the latency to begin the task. The test terminated when all 4 limbs crossed the finishing line at the other end. The cut-off duration was 2 min. A possible fall was taken as 
the maximum time. Each rat was tested 4 times with an interval of 2 minutes.

\subsubsection{Apomorphine rotational test}

The rotational performance was quantitated by a previously reported method (Roghani, Behzadi, \& Baluchnejadmojarad, 2002). In short, the rats were permitted to be adapted for 10 minutes, and complete rotations (ipsilateral and contralateral) were counted after the IP injection of the drug apomorphine hydrochloride (Sigma-Aldrich, the USA; $2 \mathrm{mg} / \mathrm{kg}$ ) in a cylindrical container (diameter $=33 \mathrm{~cm}$, height $=35 \mathrm{~cm}$ ) for $60 \mathrm{~min}$. Lastly, the pure number of done rotations was calculated.

\subsection{Oxidative stress assessment}

One week after the surgical operation, striatal and nigral tissues (left side, $n=6-7$ for each group) were punched out and made into $5 \%$ homogenates in cold lysis buffer. The final supernatant was kept at $-70^{\circ} \mathrm{C}$.

2.3.1. Determination of malondialdehyde (MDA), nitrite, ROS, the activity of catalase and superoxide dismutase (SOD), and protein

For Determining malondialdehyde (MDA) as thiobarbituric acid reactive substances or TBARS, Trichloroacetic acid (TCA) and TBARS solution were added to supernatant with incubation at boiling water for 80 minutes. After cooling, the mixture was centrifuged at $3000 \times \mathrm{g}$ for 5 minutes, and the absorbance was taken at $532 \mathrm{~nm}$ with tetraethoxypropane as its standard (Kiasalari et al., 2016).

For tissue measurement of nitrite level, Griess reagent having sulfanilamide and $\mathrm{N}$-naphthyl ethylenediamine was added, and the absorbance was taken at $540 \mathrm{~nm}$ with sodium nitrite as the standard (Kiasalari et al., 2016).

Estimation of ROS was done using a non-fluorescent lipophilic compound (dichlorofluorescein diacetate), which is converted to 2,7-dichlorofluorescein by intracellular esterase in the presence of free radicals that can cause fluorescence (Arya, Sethy, Singh, Das, \& Bhargava, 2013; Tobon-Velasco et al., 2012). The fluorescence intensity is proportional to ROS quantity. Fluorescence was read at $488 \mathrm{~nm}$ excitation and $525 \mathrm{~nm}$ emission with dichlorofluorescein itself as the standard.

SOD activity was assessed according to a past report (Mirshekar, Roghani, Khalili, Baluchnejadmojarad, \& Arab Moazzen, 2010). In short, the supernatant was incubated with a solution of xanthine and xanthine oxidase and potassium phosphate $\left(\mathrm{pH} 7.8\right.$ at $\left.37^{\circ} \mathrm{C}\right)$ for 40 minutes, and after that, nitroblue tetrazolium (NBT) dye was included. Afterward, blue formazan appearance was checked using a spectrophotometer $(550 \mathrm{~nm})$. The quantity of protein needed to inhibit NBT reduction to $50 \%$ maximum was taken as one nitrite unit of SOD activity. For activity assessment of catalase, claiborne's protocol was applied (Claiborne, 1985). In this test, $\mathrm{H}_{2} \mathrm{O}_{2}$ solution was added to a mixture of $50 \mathrm{mM}$ potassium phosphate buffer ( $\mathrm{pH} 7.0)$, and supernatant and absorbance alterations were read at $240 \mathrm{~nm}$ for 2 minutes. Bradford method was for obtaining protein quantity with bovine serum albumin as the standard (Bradford, 1976).

\subsection{Determination of DNA fragmentation}

DNA fragmentation as a reliable marker for showing apoptosis was quantitated using specific cell death detection ELISA kit (Roche Diagnostics, Germany) (Morroni et al., 2013), and its OD was reported.

2.5. Determination of nigral and striatal glial fibrillary acidic protein (GFAP), interleukin-1 $\beta$ (IL-1 $\beta$ ), inducible nitric oxide synthase (iNOS), angiotensin II (Ag II), phosphor apoptosis signalregulating kinase 1 (pASK1), and aquaporin 4

The striatal and nigral levels of these parameters were determined using sandwich enzyme-linked immunosorbent assay method and available kits according to related recommended guidelines (for pASK1 from MyBioSource Inc., San Diego, the USA, and other parameters from Cloud-Clone Corp., Houston, Texas, the USA). The absorbance of tested samples was obtained at 450 nm employing Synergy HT microplate reader (BioTek, USA). All values were obtained as $\mathrm{ng} / \mathrm{mL}$ or $\mathrm{pg} / \mathrm{mL}$ using drawn standard curves.

\subsection{Tyrosine hydroxylase (TH) immunohisto-} chemical method

Randomly selected rats ( $\mathrm{n}=5$ for each group) were anesthetized in-depth with ketamine $(150 \mathrm{mg} / \mathrm{kg})$ and perfused (transcardial method) with first normal saline and then $4 \%$ paraformaldehyde in phosphate buffer $(\mathrm{PB}$; $0.1 \mathrm{M}, \mathrm{pH} 7.4$ ). After incubation in $30 \%$ sucrose solution for $48 \mathrm{~h}, 30-\mu \mathrm{m}$ sections were prepared using a cryostat (Leica, Germany). The sections were reacted with $0.1 \%$ sodium borohydride in phosphate-buffered saline, methanol containing $0.03 \% \mathrm{H}_{2} \mathrm{O}_{2}$ and Triton X100, and bovine serum albumin in PBS. After that, the sections were reacted with primary polyclonal rabbit anti-TH antibody (Sigma-Aldrich, USA, a dilution of 1/200) in Tris-buffered saline for an overnight and then with sec- 
ondary anti-rabbit IgG-peroxidase antibody processed in goat (Sigma-Aldrich, USA,) in Tris buffer for two hours. To show the attached peroxidase, the sections were reacted with diaminobenzidine tetrahydrochloride (10 $\mathrm{mg} / 20 \mathrm{~mL}$ of tris solution and $0.03 \% \mathrm{H}_{2} \mathrm{O}_{2}$ ) for $10-12$ min, dehydrated, cleared, and coverslipped.

\subsubsection{Histological evaluation}

Counting and assessing TH-positive neuronal cells within the SNC region was conducted as stated before (Burke, Macaya, DeVivo, Kenyon, \& Janec, 1992; Roghani \& Behzadi, 2001). From the sections of SNC, a minimum of two samples showing each of 4 Paxinos and Watson planes (2.96, 3.2, 3.7, 4.2; interaural) were counted thorough assessment of the entire boundary of SNC (left side). All well-stained neuronal cells were counted. Since SNC joins Ventral Tegmental Area (VTA) at its medial border in planes 4.2 and 3.2, we took a line between the two areas by counting neurons within a grid which ran straight dorsally from the most medial portion. After observing TH-positive neurons in one area, the grid was moved further laterally. This process was completed for the entire lateral region of the SNC. At planes 3.7 and 2,96, the VTA and SNC are separated from each other. Initially, all TH-positive neurons in both SNC and SNR were taken in the counting process; in a later analysis, the isolated $\mathrm{TH}$-positive neurons in the SNR in plane 3.2 were taken separately. The number of TH-positive neurons for each nigral plane was expressed as the average of the counts obtained on the two assessed sections. The values were then added to each other for obtaining the total number of TH-positive neurons.

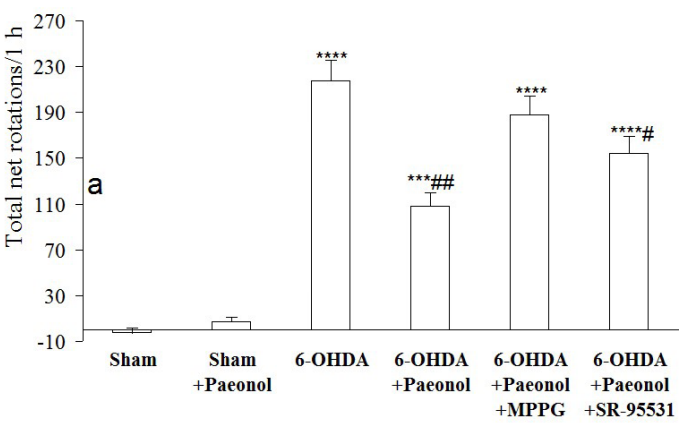

\subsection{Statistical analysis}

The obtained data were presented as Means \pm SEM. For statistical evaluation of obtained data, the parametric 1-way ANOVA test was employed with further Tukey's post-hoc test. In all analyses, the significance level was taken as less than 0.05 .

\section{Results}

\subsection{Behavioral findings}

The statistical analysis of induced rotations by apomorphine at the pre-surgery week showed no significant differences between the tested groups. However, the 6-OHDA lesioned animals on the final day of week 1 showed significant contraversive rotations compared to the sham group (an increase of $218.3 \%, \mathrm{P}<0.001$ ), and the latter rotations were significantly less in the 6-OHDA+paeonol group versus the 6-OHDA group (a decrease of $51.4 \%$, $\mathrm{P}<0.01$ ). Meanwhile, Intracerebroventricular (ICV) administration of MPPG as mGluR antagonist to a major extent or SR-95531 as GABAA receptor antagonist to a lower amount reduced the advantageous effect of paeonol in the 6-OHDA groups. This finding indicates that the beneficial effect of paeonol is further neutralized in the presence of MPPG than SR-95531 at the used doses. Furthermore, no significant alteration in rotational behavior was observed in the sham+paeonol group compared to the sham group (Figure 1A).

Evaluation of the performance of tested animals in narrow beam task revealed that the delay time to its beginning and the whole time needed for its passing (Figure 1B) were significantly greater in the 6-OHDA group

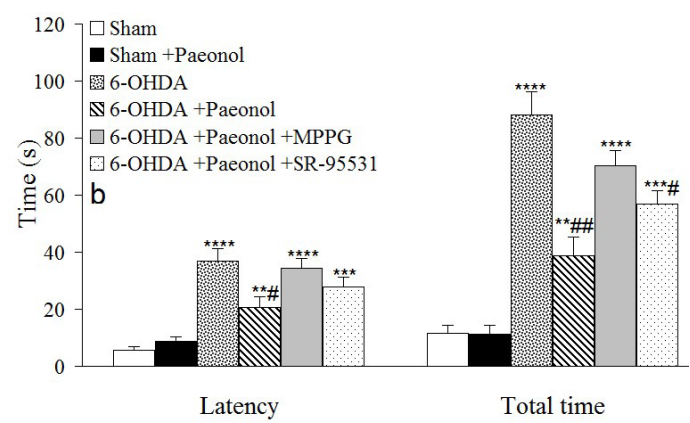

Figure 1. The effect of paeonol at a dose of $100 \mathrm{mg} / \mathrm{kg}$ on the apomorphine-produced rotational bias

A: On the latency to start the task and the whole time to perform narrow beam task; B: One week next to intrastriatal microinjection of 6-hydroxydopamine (6-OHDA).

MPPG or SR-95531 were ICV administered $30 \mathrm{~min}$ before 6-OHDA microinjection as mGluR III/II receptor and GABAA receptor antagonists, respectively. Values are expressed as Means \pm SEM; $\mathrm{n}=10-12$ for each group. ${ }^{* *} \mathrm{P}<0.01$, ${ }^{* *} \mathrm{P}<0.005 ; \mathrm{P}<0.001$ (versus sham); ${ }^{\#} \mathrm{P}<0.05,{ }^{\# \#} \mathrm{P}<0.01$ (versus 6-OHDA). 

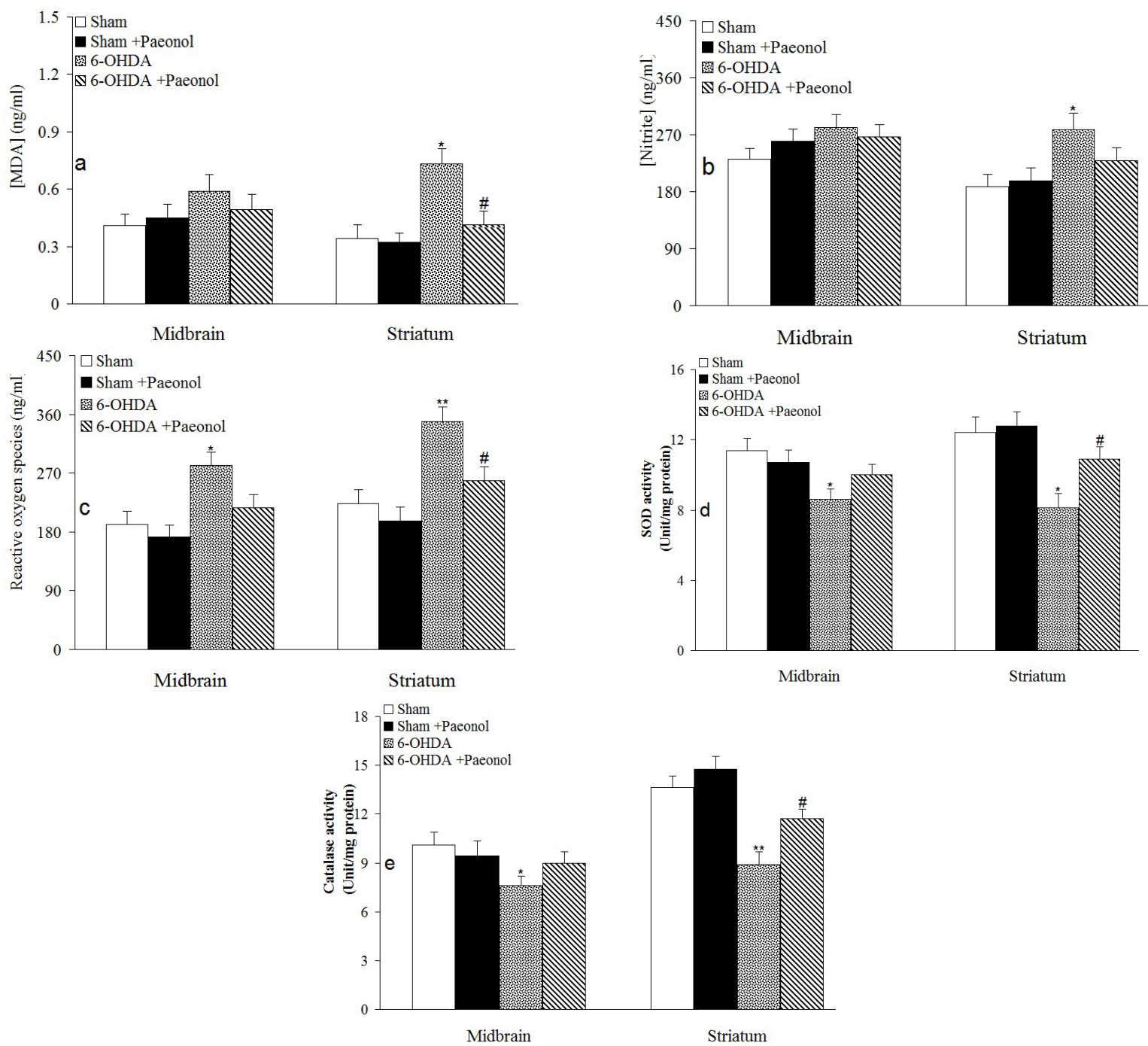

Striatum

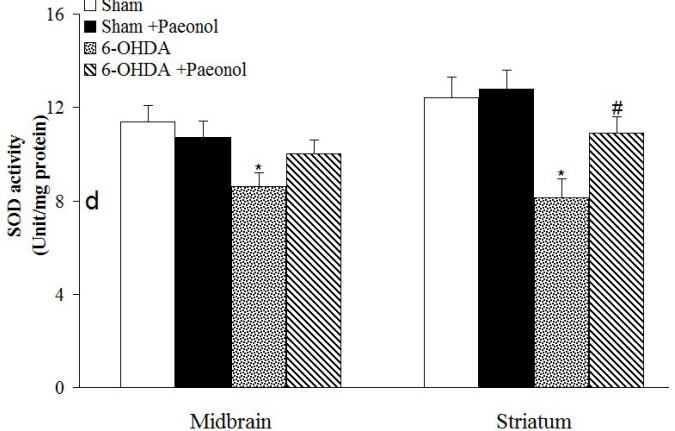

NEUR SCIENCE

Figure 2. The effect of paeonol at a dose of $100 \mathrm{mg} / \mathrm{kg}$ on nigral and striatal markers of oxidative stress event, including Malondialdehyde (MDA)

A: Nitrite; B: Reactive Oxygen Species (ROS); C: Activity of Superoxide Dismutase (SOD); D: Catalase; E: One week after intrastriatal administration of 6-hydroxydopamine (6-OHDA).

The measurements for each sample were made in duplicate. Values are expressed as Means $\pm S E M . n=7$ for each group. * $\mathrm{P}<0.05$; ** $\mathrm{P}<0.01$ (vs. Sham on the left side); ${ }^{\mathrm{P}}<0.05$ (vs. 6-OHDA on the left side)

when compared to the sham group (increases of $541.5 \%$ and $701.2 \%$, respectively, $\mathrm{P}<0.001)$ and paeonol pretreatment of the 6-OHDA group significantly reduced these times (decreases of $45.2 \%$ and $56.3 \%$, respectively, $\mathrm{P}<0.05-0.01$ ). Meanwhile, ICV treatment of MPPG to a greater extent or SR-95531 to a lower degree attenuated the observed significant beneficial effect of paeonol. This result indicates that the beneficial effect of paeonol is counteracted to a greater extent in the presence of mGluR antagonist than GABAA receptor antagonist. Besides, paeonol administration to the sham group did not cause a significant alteration of performance in narrow beam task.

\subsection{Oxidative stress findings}

Measurement of oxidative stress-indicative parameters, including tissue levels of MDA, nitrite, ROS, and activity of SOD and catalase in the midbrain and striatal areas showed that production of 6-OHDA murine model of PD caused significant elevation of striatal MDA (an increase of $114.7 \%, \mathrm{P}<0.05$ ) (Figure 2A), nitrite (an increase of $48.7 \%, \mathrm{P}<0.05$ ) (Figure 2B), nigral (an increase of $47.5 \%, \mathrm{P}<0.05$ ) and striatal (an increase of $56.5 \%$, $\mathrm{P}<0.01$ ) ROS (Figure $2 \mathrm{C}$ ) and a significantly lower activity of nigral (a decrease of $23.7 \%, \mathrm{P}<0.05$ ) and striatal (a decrease of 33.1\%, $\mathrm{P}<0.05$ ) SOD activity (Figure 2D) 

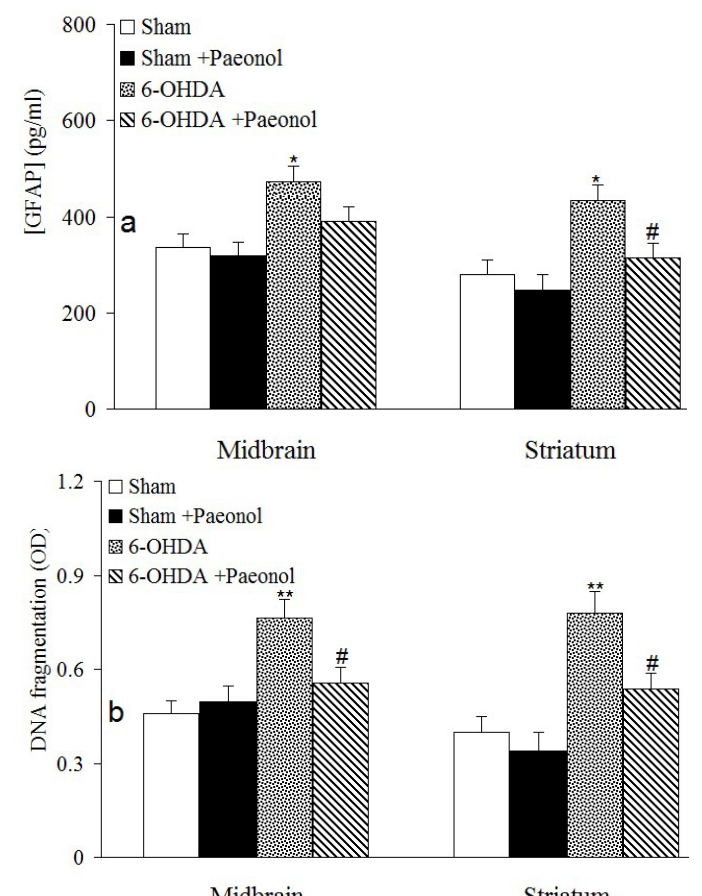

\subsection{DNA fragmentation findings}

In this research, we also quantified DNA fragmentation intensity as a reliable index of apoptosis (Figure 3B) . The achieved results indicated that the 6-OHDA group has a significantly greater quantity of nigral (an increase of $68.9 \%, \mathrm{P}<0.01$ ) and striatal (a rise of $92.5 \%, \mathrm{P}<0.01$ ) DNA fragmentation when compared to the sham group and paeonol treatment significantly and correctly attenuated this elevation in both areas, i.e. nigral (a decrease of 27.6\%, $\mathrm{P}<0.05$ ) and striatal (a decrease of $31.3 \%, \mathrm{P}<0.05$ ) tissues.

\subsection{Elisa biochemical findings}

Quantification of tissue levels of GFAP (Figure 3A) that is a specific marker of astrocytes and its elevation showing the development of astrogliosis (Filous \& Silver, 2016) indicated that its levels in nigral (an increase of $40.3 \%, \mathrm{P}<0.05$ ) and striatal (an increase of $54.7 \%$, $\mathrm{P}<0.05$ ) areas significantly escalates in the 6-OHDA group when compared to comparable data of sham group and paeonol given to the 6-OHDA group significantly and correctly reduced this abnormal increase in striatal area (a decrease of $22.7 \%, \mathrm{P}<0.05$ ).

Determination of IL-1 $\beta$ for showing the development of inflammation (Figure 3C) indicated that its nigral and striatal quantities were non-significantly raised in the 6-OHDA group when compared to the sham group and paeonol given to the 6-OHDA group non-significantly attenuated its quantity in nigral and striatal areas. Quantification of the marker iNOS (Figure 4A) indicated no significant differences between the tested groups for its nigral quantity. On the contrary, its striatal quantity significantly enhanced in the 6-OHDA group compared to the sham group (an increase of $45.2 \%, \mathrm{P}<0.05$ ), and paeonol treatment 6-OHDA-lesioned group non-significantly reduced this parameter versus the 6-OHDA group. Regarding Ag II (Figure 4B), its nigral and striatal quantity raised in the 6-OHDA group, and paeonol treatment non-significantly reduced its nigral and striatal levels. In addition, pASK1 level in nigral (an increase of $45.1 \%$, $\mathrm{P}<0.01$ ) and striatal (an increase of $77.8 \%, \mathrm{P}<0.005$ ) tissues from the 6-OHDA group increased and paeonol could significantly lower its nigral and striatal level (decreases of $25.7 \%$ and $23.1 \%$, respectively, $\mathrm{P}<0.05$ ). Furthermore, nigral (an increase of 79.4\%, $\mathrm{P}<0.01$ ) and striatal (an increase of $47.3 \%, \mathrm{P}<0.05$ ) level of aquaporin 4 raised in the 6-OHDA group and paeonol pretreatment significantly and properly reduced its nigral level (a decrease of $33.5 \%, \mathrm{P}<0.05$ ). 

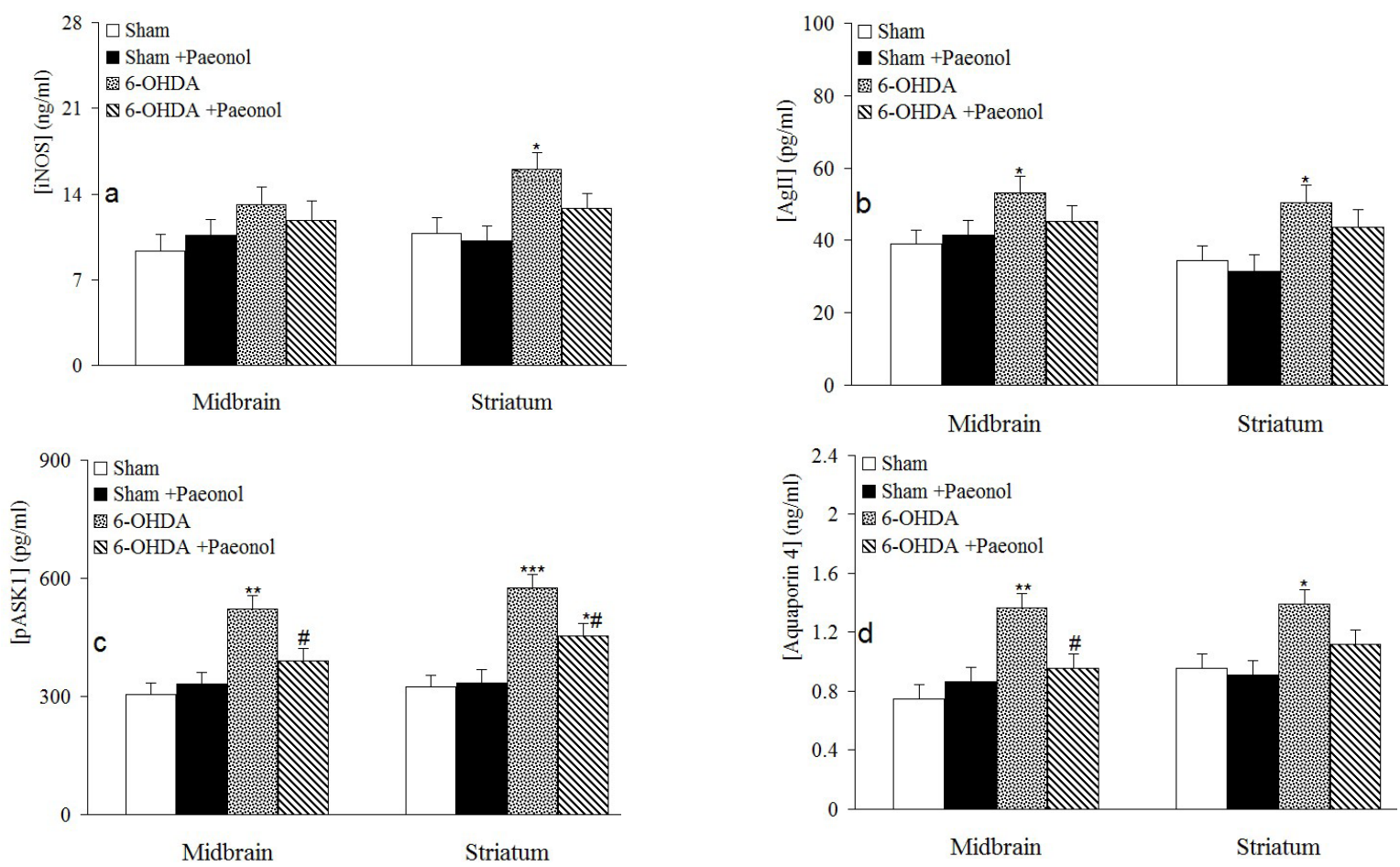

Figure 4. The effect of paeonol at a dose of $100 \mathrm{mg} / \mathrm{kg}$ on nigral and striatal quantities of iNOS

A: Angiotensin II (Ag II); B: Phosphor apoptosis signal-regulating kinase 1 (pASK1); C: Aquaporin 4; D: One week after intrastriatal administration of 6-hydroxydopamine (6-OHDA).

The measurements were made in duplicate for samples. Values are expressed as Means $\pm S E M$. $n=5$ for each group; * $P<0.05$; ${ }^{* *} \mathrm{P}<0.01$; ${ }^{* * *} \mathrm{P}<0.005$ (vs. sham on left side); ${ }^{\mathrm{P}}<0.05$ (vs. 6-OHDA on left side).

3.5. Tyrosine Hydroxylase (TH) immunohistochemistry

TH immunohistochemical assessment (Figure 5) revealed no significant and notable difference between the tested groups, i.e. the sham and sham+paeonol groups, for TH-positive neurons on the left side of SNC. However, a significant decline of TH-containing neurons was noted in the 6-OHDA group (a decrease of $42.5 \%$, $\mathrm{P}<0.005)$ compared to the sham group. Additionally, a less significant reduction of TH-containing neurons was noted in the 6OHDA+paeonol group (a decrease of $21.5 \%, \mathrm{P}<0.05$ ) compared to the 6-OHDA group. Furthermore, the number of TH-immunoreactive neurons on the left side of SNC was significantly more in the 6-OHDA+paeonol group as compared to 6-OHDA animals (an increase of $37.4 \%, \mathrm{P}<0.05$ ).

\section{Discussion}

In this study, paeonol pretreatment of the 6-OHDAlesioned rats alleviated drug-induced motor asymmetry and improved their performance in narrow beam task via amelioration of oxidative stress, apoptosis, and as- trogliosis intensity, and its beneficial effect is somewhat dependent on pASK1/mGluR III/GABAA signaling.

Unilateral injection of the specific catecholaminergic neurotoxin 6-OHDA into the striatum produces a reproducible and valid model of PD in the rat that is suitable for exploration of molecular mechanisms involved in degeneration of mesencephalic dopaminergic neurons and to assess the beneficial potency of newly-found chemical agents (Kirik, Rosenblad, \& Bjorklund, 1998; Negahdar et al., 2015). Microinjection of 6-OHDA into the nigrostriatal dopaminergic system finally reduces dopamine quantity on the injected side and concomitant increase in the density of postsynaptic dopaminergic receptors (Cass \& Peters, 2010), with the development of a motor asymmetry, as shown by systemic administration of direct-acting dopaminergic receptors agonists like apomorphine (Roghani et al., 2002; Schwarting \& Huston, 1997). A similar finding was also obtained in our study, as demonstrated by contraversive rotations following apomorphine in the 6-OHDA-lesioned group. Since PO administration of paeonol lowered apomorphineinduced rotations in our research, this fact obviously and indirectly indicated its ability to retard and or stop 

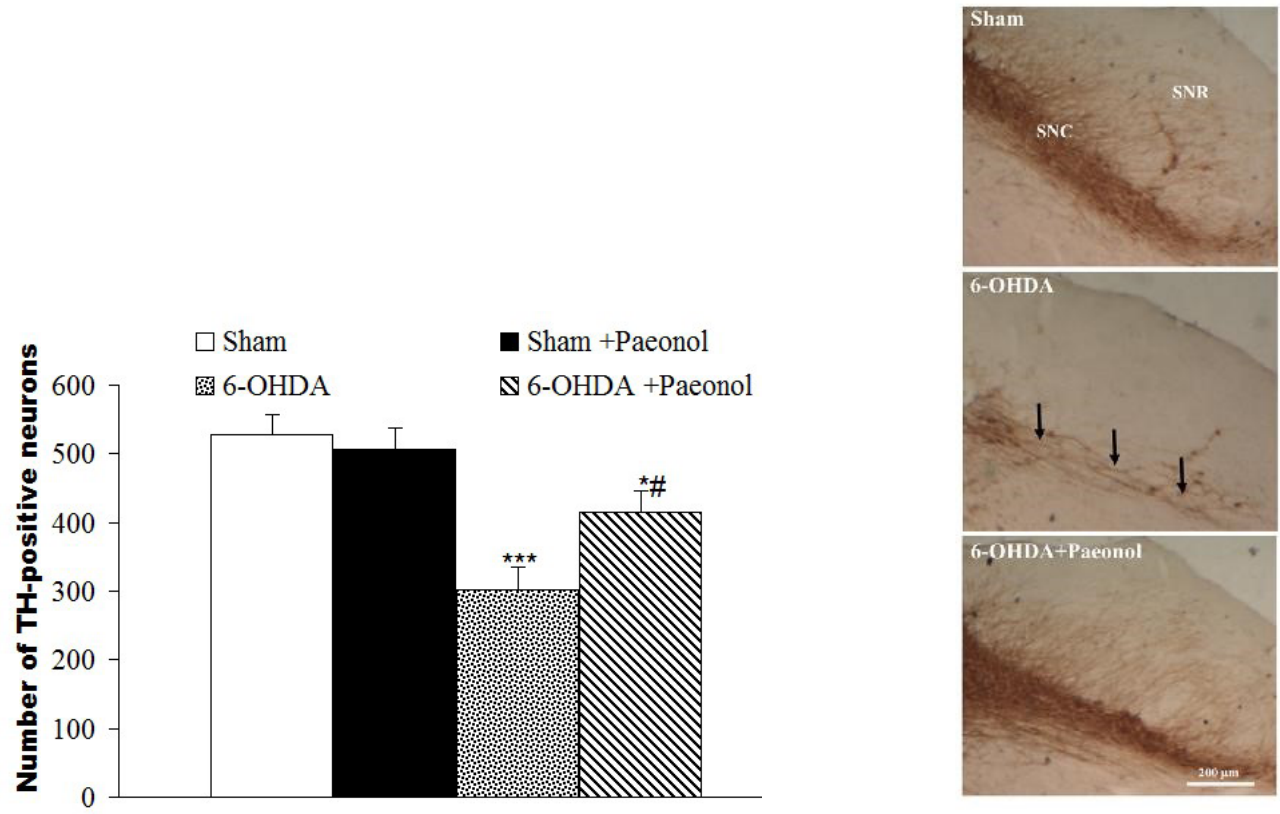

NEUR SCIENCE

Figure 5. The effect of paeonol at a dose of $100 \mathrm{mg} / \mathrm{kg}$ on the average number of Tyrosine Hydroxylase (TH)-containing neurons of the left side of substantia nigra pars compacta (SNC)

A: Photomicrographs obtained from midbrain coronal sections one week after intrastriatal administration of 6-hydroxydopamine (6-OHDA). Arrows indicate an area of remarkable loss of TH-containing neurons.

Values are expressed as means \pm SEM. $\mathrm{n}=4-5$ for each group. ( $\mathrm{SNR}=$ Substantia nigra pars reticulate); $\mathrm{P}<0.05$; ** $\mathrm{P}<0.01$ (versus Sham); ${ }^{~} \mathrm{P}<0.05$ (versus 6-OHDA).

reduction of the striatal quantity of dopamine. Besides, the 6-OHDA-lesioned group also displayed perceptible and notable motor derangement in the narrow beam paradigm, as shown by an elevated latency to start the task and with a longer time required for beam passing. This abnormal behavior in narrow beam task in the rats following 6-OHDA lesioning is mediated through reduction of dopamine quantity in the damaged striatum with ensuing development of bradykinesia (Allbutt \& Henderson, 2007). This motor abnormality in the 6-OHDA group ameliorated following oral administration of paeonol, denoting its power in attenuating damage of midbrain dopaminergic neurons and preservation of dopamine quantity at appropriate levels on the damaged side.

Tyrosine Hydroxylase (TH) enzyme is the key and rate-limiting element for catalyzing biosynthesis of catecholamines such as dopamine. Following striatal microinjection of the neurotoxin 6-OHDA, progressive neurodegeneration and demise of TH-containing neurons occur in midbrain SNC (Macchi et al., 2015). In our study, the number of TH-positive neurons was greater in the paeonol-pretreated 6-OHDA group when compared to the non-treated lesioned group. This result suggests its protective effect against 6-OHDA neurotoxicity. When cells are damaged by the neurotoxin 6-OHDA, the level of apoptosis enhances (Hwang \& Chun, 2012). In this research, oral administration of paeonol could attenuate DNA fragmentation as a reliable indicator of an apoptotic event and, in this way to diminish degeneration of nigrostriatal dopaminergic neuronal cells in midbrain SNC. Similar to our findings, a previous study has also shown that oral administration of paeonol could dosedependently attenuate apoptosis of neurons and caspase 3 expression in the streptozotocin-induced model of diabetic encephalopathy (Liu et al., 2013).

Oxidative stress enhancement is another crucial factor in the neurodegeneration of the nigrostriatal dopaminergic system in PD (Jiang, Sun, \& Chen, 2016). In this study, striatal level of MDA as a consistent marker of lipid peroxidation and nitrite as a metabolite of nitric oxide estimated level of ROS in both nigral and striatal lysates increased, and the activity of the defensive enzymes, including catalase and SOD, reduced in the 6-OHDA-lesioned group, implying a state of oxidative stress development in this murine model of PD. The importance of oxidative stress in the occurrence of PD has already been reported, as reviewed in previous reports (Jiang et al., 2016; Sarrafchi, Bahmani, Shirzad, \& Rafieian-Kopaei, 2016). Interestingly, oral administration of paeonol ameliorated MDA and ROS and improved activity of catalase and SOD in 
the 6-OHDA lesioned group. In other words, paeonol has attenuated oxidative stress following 6-OHDA. In support of our findings, it has been shown that paeonol could exert therapeutic effects in the methyl4-phenyl-1,2,3,6tetrahydropyridine (MPTP)/probenecid-produced murine model of $\mathrm{PD}$ via decreasing the damage of oxidative stress burden and neuroinflammation and also by raising neurotrophic effects on dopaminergic neurons (Shi et al., 2016). Additionally, paeonol is considered a neuroprotective chemical mediated by lowering microglia-mediated inflammation- and oxidative stress-produced neuronal damage (Tseng, Hsu, Shih, \& Lo, 2012).

In our study, the 6-OHDA-lesioned animals exhibited an elevated level of nigral and striatal GFAP, strongly demonstrating the appearance of astrogliosis and neuroinflammation in the nigrostriatal dopaminergic system that is also reported in an earlier investigation (Calou et al., 2014). In contrast, paeonol lowered GFAP overexpression in both nigral and striatal tissues that may be ascribed to its inflammation suppressing property (Lou et al., 2017). However, there is no unified report on the possible effect of paeonol on GFAP and or astrogliosis in the literature.

In our study, nigral and striatal level of IL- $1 \beta$ as a specific marker of inflammation and striatal level of iNOS as an inflammation-related marker non-significantly increased in the 6-OHDA group after one week. In this regard, it has been shown that microglia quickly adopt a reactive condition in the substantia nigra and neostriatum following microinjection of 6-OHDA into the medial forebrain bundle that is associated with overexpression and or overproduction of pro-inflammatory markers such as Tumor Necrosis Factor-Alpha (TNF $\alpha$ ) and iNOS that are involved in neurodegeneration of dopaminergic neurons (Haas et al., 2016). Contrary to our findings of inflammatory markers, researchers have shown an increased level of inflammatory cytokines like TNF $\alpha$ and IL-1 $\beta$ in the 6-OHDA model of PD (Kheradmand, Nayebi, Jorjani, Khalifeh, \& Haddadi, 2016). The main reason for this discrepancy is that inflammatory cytokines are usually upregulated during the first hours and at most the early days of lesion induction by the neurotoxins, and their level drops during the next days. In support of this idea, Haas et al. have shown that inflammatory markers like TNF- $\alpha$ and iNOS increase in the 6-hydroxydopamine mouse model of PD during the first 2 days after lesioning, and their level returns to baseline level (Haas et al., 2016).

$\mathrm{Ag}$ II is involved in the progression of inflammation and oxidative stress in PD, and its elevation increases the degeneration process in dopaminergic neurons (Rodri-
guez-Pallares et al., 2008). In our study, one week after striatal 6-OHDA, the nigral and striatal levels of Ag II significantly increased. However, its level did not significantly reduce in the presence of paeonol. A relation between overexpression of pASK1 and 6-OHDA-induced dopaminergic neurodegeneration ( $\mathrm{Hu}$ et al., 2011) and between aquaporin $4 \mathrm{mRNA}$ expression and

lesioned substantia nigra due to 6-OHDA (Vizuete et al., 1999) has already been established. (It was also found out in this study.) Although oral administration of paeonol could lower nigral and striatal level of pASK1 and nigral level of aquaporin 4 in the 6-OHDA-lesioned group; however, there is still no definite experimental report in this respect.

The beneficial effect of oral paeonol in the 6-OHDA-lesioned group was ameliorated following ICV application of mGluR III receptor antagonist MPPG to a great extent or GABAA receptor antagonist SR-95531 to a smaller intensity. This indicates the key role of mGluR III and GABAA receptor cascade in producing the protective effect of paeonol. Consistent with our findings, it has found out that the neuroprotective effect of paeonol is, to some extent, dependent on its ability to lower glutamate neurotoxicity and suppresses apoptosis (Wang et al., 2011). Since a combination of mGluR III agonist and mGluR I antagonist produces notable nigrostriatal neuroprotection in the 6-OHDA murine model of PD (Vernon et al., 2008); therefore, it is logical that after application of MPPG, the neuroprotective effect of paeonol to be attenuated. Besides, GABAA activation is associated with neuroprotective effects in hemiparkinsonian rats (Yunes et al., 2015), and paeonol has been shown to affect GABAA receptors (Sun et al., 2011). Thus, it is expected that following SR-95531, the neuroprotective effect of paeonol to be reduced, as was observed in our study.

On the whole, our findings indicated the neuroprotective property of paeonol in the 6-OHDA murine model of PD through alleviation of oxidative stress, apoptosis, astrogliosis, and its effect is somewhat dependent on pASK1/mGluR III/GABAA signaling.

\section{Ethical Considerations}

\section{Compliance with ethical guidelines}

Animal procedures in this research were done in compliance with the Guide for the Care and Use of Laboratory Animals, specified by the National Institutes of Health (NIH) of the USA and certified by the local Animal Ethics Committee of Iran University of Medical Sciences (Tehran, Iran) in 2016. 
Funding

This paper was extracted from $\mathrm{PhD}$. dissertation project of Jamileh Ghalami, Department of Physiology, Faculty of Medicine, Iran University of Medical Sciences.

\section{Authors' contributions}

Conceptualization, data collection, and writing - original draft: Jamileh Ghalami and Monireh Mansouri; Data analysis, writing - review \& editing, Investigation, and Supervision: Safoura Khamse, Tourandokht Baluchnejadmojarad, and Mehrdad Roghani.

\section{Conflict of interest}

The authors declared no conflict of interest.

\section{References}

Ahmad, M., Saleem, S., Ahmad, A. S., Yousuf, S., Ansari, M. A., \& Khan, M. B., et al. (2005). Ginkgo biloba affords dose-dependent protection against 6-hydroxydopamineinduced parkinsonism in rats: Neurobehavioural, neurochemical and immunohistochemical evidences. Journal of Neurochemistry, 93(1), 94-104. [DOI:10.1111/j.1471-4159.2005.03000.x] [PMID]

Allbutt, H. N., \& Henderson, J. M. (2007). Use of the narrow beam test in the rat, 6hydroxydopamine model of Parkinson's disease. Journal of Neuroscience Methods, 159(2), 195-202. [DOI:10.1016/j. jneumeth.2006.07.006] [PMID]

Arya, A., Sethy, N. K., Singh, S. K., Das, M., \& Bhargava, K. (2013) Cerium oxide nanoparticles protect rodent lungs from hypobaric hypoxia-induced oxidative stress and inflammation. International Journal of Nanomedicine, 8, 4507-20. [DOI:10.2147/IJN. S53032] [PMID] [PMCID]

Beitz, J. M. (2014). Parkinson's disease: A review. Front Biosci (Schol Ed), 6, 65-74. [DOI:10.2741/S415] [PMID]

Braak, H., Ghebremedhin, E., Rub, U., Bratzke, H., \& Del Tredici, K. (2004). Stages in the development of Parkinson's disease-related pathology. Cell and Tissue Research, 318(1), 121134. [DOI:10.1007/ s00441-004-0956-9] [PMID]

Bradford, M. M. (1976). A rapid and sensitive method for the quantitation of microgram quantities of protein utilizing the principle of protein-dye binding. Analytical Biochemistry, 72, 248-254 [DOI:10.1016/0003-2697(76)90527-3]

Burke, R. E., Macaya, A., DeVivo, D., Kenyon, N., \& Janec, E. M (1992). Neonatal hypoxicischemic or excitotoxic striatal injury results in a decreased adult number of substantia nigra neurons. Neuroscience, 50(3), 559-69. [DOI:10.1016/0306-4522(92)90447-A]

Calou, I., Bandeira, M. A., Aguiar-Galvao, W., Cerqueira, G., Siqueira, R., \& Neves, K. R., et al. (2014). Neuroprotective Properties of a Standardized Extract from Myracrodruon urundeuva Fr. All. (Aroeira-Do-Sertao), as Evaluated by a
Parkinson's Disease Model in Rats. Parkinson's Disease, 2014 519615. [DOI:10.1155/2014/519615] [PMID] [PMCID]

Cass, W. A., \& Peters, L. E. (2010). Neurturin protects against 6-hydroxydopamine-induced reductions in evoked dopamine overflow in rat striatum. Neurochemistry International, 57(5), 540-6. [DOI:10.1016/j.neuint.2010.06.019] [PMID] [PMCID]

Choy, K. W., Lau, Y. S., Murugan, D., \& Mustafa, M. R. (2017) Chronic treatment with paeonol improves endothelial function in mice through inhibition of endoplasmic reticulum stress-mediated oxidative stress. PLoS One, 12(5), e0178365. [DOI:10.1371/journal.pone.0178365] [PMID] [PMCID]

Claiborne, A. (1985). Catalase activity. In: CRC Handbook of Methods for Oxygen Radical Research, edited by Greenwald RA. Boca Raton, Florida: Routledge \& CRC Press, 283-284.

Dauer, W, \& Przedborski, S. (2003). Parkinson's Disease. Neuron 39(6), 889-909. [DOI:10.1016/S0896-6273(03)00568-3]

Filous, A. R., \& Silver, J. (2016). “Targeting astrocytes in CNS injury and disease: A translational research approach". Prog Neurobiol, 144, 173-187. doi:10.1016/j.pneurobio.2016.03.009 [DOI:10.1016/j.pneurobio.2016.03.009] [PMID] [PMCID]

Gustot, A., Gallea, J. I., Sarroukh, R., Celej, M. S., Ruysschaert, J. M., \& Raussens, V. (2015). Amyloid fibrils are the molecular trigger of inflammation in Parkinson's disease. Biochemical Journal, 471(3), 323-333. [DOI:10.1042/BJ20150617] [PMID]

Haas, S. J., Zhou, X., Machado, V., Wree, A., Krieglstein, K., \& Spittau, B. (2016). Expression of Tgfbeta1 and Inflammatory Markers in the 6-hydroxydopamine Mouse Model of Parkinson's Disease. Frontiers in Molecular Neuroscience, 9, 7. [DOI:10.3389/fnmol.2016.00007] [PMID] [PMCID]

He, X., Cai, Q., Li, J., \& Guo, W. (2018). Involvement of braingut axis in treatment of cerebral infarction by beta-asaron and paeonol. Neuroscience Letters, 666, 78-84. [DOI:10.1016/j.neulet.2017.12.036] [PMID]

Hornykiewicz, O, \& Kish, SJ. (1987). Biochemical pathophysiology of Parkinson's Disease. Advances in Neurology, 45, 19-34 https://ci.nii.ac.jp/naid/10007515684/

Hu, J., Li, Y. L., Li, Z. L., Li, H., Zhou, X. X., \& Qiu, P. C., et al. (2012). Chronic supplementation of paeonol combined with danshensu for the improvement of vascular reactivity in the cerebral basilar artery of diabetic rats. International Journal of Molecular Sciences, 13(11), 1456514578. [DOI:10.3390/ ijms131114565] [PMID] [PMCID]

Hu, X., Weng, Z., Chu, C. T., Zhang, L., Cao, G., \& Gao, Y., etal (2011). Peroxiredoxin2 protects against 6-hydroxydopamineinduced dopaminergic neurodegeneration via attenuation of the Apoptosis Signal-Regulating Kinase (ASK1) signaling cascade. Journal of Neuroscience, 31(1), 247-61. [DOI:10.1523/ JNEUROSCI.4589-10.2011] [PMID] [PMCID]

Hwang, C. K., \& Chun, H. S. (2012). Isoliquiritigenin isolated from licorice Glycyrrhiza uralensis prevents 6-hydroxydopamine-induced apoptosis in dopaminergic neurons. Bioscience, Biotechnology, and Biochemistry, 76(3), 536-43. [DOI:10.1271/ bbb.110842] [PMID]

Jiang, T., Sun, Q., \& Chen, S. (2016). Oxidative stress: A major pathogenesis and potential therapeutic target of antioxidative agents in Parkinson's disease and Alzheimer's Disease. Progress in Neurobiology, 147, 1-19. [DOI:10.1016/j.pneurobio.2016.07.005] [PMID] 
Kheradmand, A., Nayebi, A. M., Jorjani, M., Khalifeh, S., \& Haddadi, R. (2016). Effects of WR1065 on 6-hydroxydopamine-induced motor imbalance: Possible involvement of oxidative stress and inflammatory cytokines. Neuroscience Letters, 627, 7-12. [DOI:10.1016/j.neulet.2016.05.040] [PMID]

Kiasalari, Z., Baluchnejadmojarad, T., \& Roghani, M. (2016). Hypericum perforatum hydroalcoholic extract mitigates motor dysfunction and is neuroprotective in intrastriatal 6-Hydroxydopamine Rat Model of Parkinson's Disease. Cellular and Molecular Neurobiology, 36(4), 521-30. [DOI:10.1007/ s10571-015-0230-6] [PMID]

Kiasalari, Z., Khalili, M., Baluchnejadmojarad, T., \& Roghani, M. (2016). Protective effect of oral hesperetin against unilateral striatal 6-hydroxydopamine damage in the rat. Neurochemical Research, 41(5), 1065-72. [DOI:10.1007/s11064-015-1796-6] [PMID]

Kirik, D., Rosenblad, C., \& Bjorklund, A. (1998). Characterization of behavioral and neurodegenerative changes following partial lesions of the nigrostriatal dopamine system induced by intrastriatal 6-hydroxydopamine in the rat. Experimental Neurology, 152(2), 259277. [DOI:10.1006/exnr.1998.6848] [PMID]

Lau, C. H., Chan, C. M., Chan, Y. W., Lau, K. M., Lau, T. W.,\& Lam, F. C., et al. (2007). Pharmacological investigations of the anti-diabetic effect of Cortex Moutan and its active component paeonol. Phytomedicine, 14(11), 778-84. [DOI:10.1016/j. phymed.2007.01.007] [PMID]

Liao, W. Y., Tsai, T. H., Ho, T. Y., Lin, Y. W., Cheng, C. Y., \& Hsieh, C. L. (2016). Neuroprotective effect of paeonol mediates antiinflammation via suppressing toll-like receptor 2 and toll-like receptor 4 signaling pathways in cerebral ischemia-reperfusion injured rats. Evidence-Based Complementary and Alternative Medicine, 2016, 3704647. [DOI:10.1155/2016/3704647] [PMID] [PMCID]

Liu, J., Feng, L., Ma, D., Zhang, M., Gu, J., \& Wang, S., et al. (2013). Neuroprotective effect of paeonol on cognition deficits of diabetic encephalopathy in streptozotocininduced diabetic rat. Neuroscience Letters, 549, 63-8. [DOI:10.1016/j. neulet.2013.06.002] [PMID]

Liu, J., Wang, S., Feng, L., Ma, D., Fu, Q., \& Song, Y., et al. (2013). Hypoglycemic and antioxidant activities of paeonol and its beneficial effect on diabetic encephalopathy in streptozotocininduced diabetic rats. Journal of Medicinal Food, 16(7), 577-86. [DOI:10.1089/jmf.2012.2654] [PMID]

Liu, N., Feng, X., Wang, W., Zhao, X., \& Li, X. (2017). Paeonol protects against TNF-alphainduced proliferation and cytokine release of rheumatoid arthritis fibroblast-like synoviocytes by upregulating FOXO3 through inhibition of miR-155 expression. Inflammation Research, 66(7), 603-10. [DOI:10.1007/ s00011-017-1041-7] [PMID]

Lou, Y., Wang, C., Tang, Q., Zheng, W., Feng, Z., \& Yu, X., et al. (2017). Paeonol Inhibits IL-1beta-Induced Inflammation via PI3K/Akt/NF-kappaB Pathways: In vivo and vitro studies. Inflammation, 40(5), 1698-706. [DOI:10.1007/s10753-017-06118] [PMID]

Macchi, B., Di Paola, R., Marino-Merlo, F., Felice, M. R., Cuzzocrea, S., \& Mastino, A. (2015). Inflammatory and cell death pathways in brain and peripheral blood in Parkinson's disease. CNS \& Neurological Disorders-Drug Targets, 14(3), 313324. [DOI:10.2174/1871527314666150225124928] [PMID]
Mirshekar, M., Roghani, M., Khalili, M., Baluchnejadmojarad T., \& Arab Moazzen, S. (2010). Chronic oral pelargonidin alleviates streptozotocin-induced diabetic neuropathic hyperalgesia in rat: Involvement of oxidative stress. Iranian Biomedical Journal, 14(1-2), 33-9. [PMCID] [PMID]

Morroni, F., Tarozzi, A., Sita, G., Bolondi, C., Zolezzi Moraga, J. M., Cantelli-Forti, G., \& Hrelia, P. (2013). Neuroprotective effect of sulforaphane in 6-hydroxydopaminelesioned mouse model of Parkinson's disease. Neurotoxicology, 36, 63-71. [DOI:10.1016/j.neuro.2013.03.004] [PMID]

Negahdar, F., Mehdizadeh, M., Joghataei, M. T., Roghani, M., Mehraeen, F., \& Poorghayoomi, E. (2015). Berberine chloride pretreatment exhibits neuroprotective effect against 6hydroxydopamine-induced neuronal insult in rat. Iranian Journal of Pharmaceutical Research, 14(4), 1145-52. [PMID]

Ouyang, M., \& Shen, X. (2006). Critical role of ASK1 in the 6-hydroxydopamine-induced apoptosis in human neuroblastoma SH-SY5Y cells. Journal of Neurochemistry, 97(1), 234-44. [DOI:10.1111/j.1471-4159.2006.03730.x] [PMID]

Paxinos, G., \& Watson, C. (1986). The rat brain in stereotaxic coordinates $2^{\text {nd }} e d$, Academic Press, San Diego.

Ping, M., Xiao, W., Mo, L., Xiao, X., Song, S., \& Tang, W., et al. (2014). Paeonol attenuates advanced oxidation protein product-induced oxidative stress injury in THP- 1 macrophages. Pharmacology, 93(5-6), 286-95. [DOI:10.1159/000363577] [PMID]

Prusty, S. K., Sahu, P. K., \& Subudhi, B. B. (2017). Angiotensin mediated oxidative stress and neuroprotective potential of antioxidants and AT1 receptor blockers. Mini-Reviews in Medicinal Chemistry, 17(6), 518-28. [DOI:10.2174/13895575166661 61025094539] [PMID]

Ray, A., Sehgal, N., Karunakaran, S., Rangarajan, G., \& Ravindranath, V. (2015). MPTP activates ASK1-p38 MAPK signaling pathway through TNF-dependent Trx1 oxidation in parkinsonism mouse model. Free Radical Biology and Medicine, 87, 312-25. [DOI:10.1016/j.freeradbiomed.2015.06.041] [PMID]

Ren, W. Q., Tian, Z. M., Yin, F., Sun, J. Z., \& Zhang, J. N. (2016) Extracellular alphasynuclein--a possible initiator of inflammation in Parkinson's disease. Pharmazie, 71(2), 51-55.

Rodriguez-Pallares, J., Rey, P., Parga, J. A., Munoz, A., Guerra, M. J., \& Labandeira-Garcia, J. L. (2008). Brain angiotensin enhances dopaminergic cell death via microglial activation and NADPH-derived ROS. Neurobiology of Disease, 31(1), 58-73. [DOI:10.1016/j.nbd.2008.03.003] [PMID]

Roghani, M., \& Behzadi, G. (2001). Neuroprotective effect of vitamin E on the early model of Parkinson's disease in rat: Behavioral and histochemical evidence. Brain Research, 892(1), 211-7. [DOI:10.1016/S0006-8993(00)03296-0]

Roghani, M., Behzadi, G., \& Baluchnejadmojarad, T. (2002). Efficacy of elevated body swing test in the early model of Parkinson's disease in rat. Physiology and Behavior, 76(4-5), 507-10. [DOI:10.1016/S0031-9384(02)00753-9]

Sarrafchi, A., Bahmani, M., Shirzad, H., \& Rafieian-Kopaei, M. (2016). Oxidative stress and Parkinson's disease: New hopes in treatment with herbal antioxidants. Current Pharmaceutical Design, 22(2), 238-246. [DOI:10.2174/138161282266615111215 1653] [PMID] 
Schwarting, R. K., \& Huston, J. P. (1997). Behavioral and neurochemical dynamics of neurotoxic meso-striatal dopamine lesions. Neurotoxicology, 18(3), 689-708.

Shi, X., Chen, Y. H., Liu, H., \& Qu, H. D. (2016). Therapeutic effects of paeonol on methyl4-phenyl-1,2,3,6-tetrahydropyridine/probenecid-induced Parkinson's disease in mice. Molecular Medicine Reports, 14(3), 2397-404. [DOI:10.3892/ mmr.2016.5573] [PMID] [PMCID]

Spittau, B. (2015). Transforming growth factor beta1-mediated anti-inflammation slows progression of midbrain dopaminergic neurodegeneration in Parkinson's disease? Neural Regeneration Research, 10(10), 1578-80. [DOI:10.4103/16735374.165228] [PMID] [PMCID]

Srivastav, S., Fatima, M., \& Mondal, A. C. (2017). Important medicinal herbs in Parkinson's disease pharmacotherapy. Biomedicine \& Pharmacotherapy, 92, 856-63. [DOI:10.1016/j.biopha.2017.05.137] [PMID]

Sun, H., Liang, R., Yang, B., Zhou, Y., Liu, M., Fang, F., ... Hu, G. (2016). Aquaporin-4 mediates communication between astrocyte and microglia: Implications of neuroinflammation in experimental Parkinson's disease. Neuroscience, 317, 65-75. [DOI:10.1016/j.neuroscience.2016.01.003] [PMID]

Sun, S., Qiao, M., \& Zhang, H. (2011). [Treatment of premenstrual syndrome with Chinese medicine formula, baixiangdan capsule: implications for neuron viability and GABA(A) receptor modulation in rat cortex]. Zhongguo Zhong Yao Za Zhi. Zhongguo Zhongyao Zazhi. China Journal of Chinese Materia Medica, 36(11), 1508-1511. [PMID]

Sun, X. L., Chen, B. Y., Zhao, H. K., Cheng, Y. Y., Zheng, M. H., \& Duan, L., et al. (2016). Gas1 up-regulation is inducible and contributes to cell apoptosis in reactive astrocytes in the substantia nigra of LPS and MPTP models. Journal of Neuroinflammation, 13(1), 180. [DOI:10.1186/s12974-016-0643-2] [PMID] [PMCID]

Tao, W., Wang, H., Su, Q., Chen, Y., Xue, W., \& Xia, B., et al. (2016). Paeonol attenuates lipopolysaccharide-induced depressive-like behavior in mice. Psychiatry Research, 238, 116121. [DOI:10.1016/j.psychres.2016.02.033] [PMID]

Tobon-Velasco, J. C., Vazquez-Victorio, G., Macias-Silva, M., Cuevas, E., Ali, S. F.,\& Maldonado, P. D., et al. (2012). S-allyl cysteine protects against 6hydroxydopamine-induced neurotoxicity in the rat striatum: Involvement of Nrf2 transcription factor activation and modulation of signaling kinase cascades. Free Radical Biology and Medicine, 53(5), 1024-40. [DOI:10.1016/j.freeradbiomed.2012.06.040] [PMID]

Tseng, Y. T., Hsu, Y. Y., Shih, Y. T., \& Lo, Y. C. (2012). Paeonol attenuates microgliamediated inflammation and oxidative stress-induced neurotoxicity in rat primary microglia and cortical neurons. Shock, 37(3), 312-8. [DOI:10.1097/ SHK.0b013e31823fe939] [PMID]

Vernon, A. C., Croucher, M. J., \& Dexter, D. T. (2008). Additive neuroprotection by metabotropic glutamate receptor subtype-selective ligands in a rat Parkinson's model. Neuroreport, 19(4), 475-8. [DOI:10.1097/WNR.0b013e3282f602df] [PMID]

Vizuete, M. L., Venero, J. L., Vargas, C., Ilundain, A. A., Echevarria, M., \& Machado, A., et al. (1999). Differential upregulation of aquaporin- 4 mRNA expression in reactive astrocytes after brain injury: Potential role in brain edema. Neurobiology of Disease, 6(4), 245258. [DOI:10.1006/nbdi.1999.0246] [PMID]
Wang, X., Zhu, G., Yang, S., Wang, X., Cheng, H., \& Wang, F., et al. (2011). Paeonol prevents excitotoxicity in rat pheochromocytoma PC12 cells via downregulation of ERK activation and inhibition of apoptosis. Planta Medica, 77(15), 1695-701. [DOI:10.1055/s-0030-1271033] [PMID]

Wu, S. H., Wu, D. G., \& Chen, Y. W. (2010). Chemical constituents and bioactivities of plants from the genus Paeonia. Chemistry \& Biodiversity, 7(1), 90-104. [DOI:10.1002/cbdv.200800148] [PMID]

Yunes, R., Casas, S., Gaglio, E., \& Cabrera, R. (2015). Progesterone exerts a neuromodulatory effect on turning behavior of hemiparkinsonian male rats: Expression of 3 alpha hydroxysteroid oxidoreductase and allopregnanolone as suggestive of GABAA receptors involvement. Parkinson's Disease, 2015, 431690. [DOI:10.1155/2015/431690] [PMID] [PMCID]

Zhang, J., Yang, B., Sun, H., Zhou, Y., Liu, M., \& Ding, J., et al. (2016). Aquaporin-4 deficiency diminishes the differential degeneration of midbrain dopaminergic neurons in experimental Parkinson's disease. Neuroscience Letters, 614, 7-15. [DOI:10.1016/j.neulet.2015.12.057] [PMID]

Zhao, B., Shi, Q. J., Zhang, Z. Z., Wang, S. Y., Wang, X., \& Wang, H. (2018). Protective effects of paeonol on subacute/chronic brain injury during cerebral ischemia in rats. Experimental and Therapeutic Medicine, 15(4), 3836-46. [DOI:10.3892/etm.2018.5893]

Zhong, S. Z., Ge, Q. H., Qu, R., Li, Q., \& Ma, S. P. (2009). Paeonol attenuates neurotoxicity and ameliorates cognitive impairment induced by d-galactose in ICR mice. Journal of the Neurological Sciences, 277(1-2), 58-64. [DOI:10.1016/j.jns.2008.10.008] [PMID]

Zhou, J., Zhou, L., Hou, D., Tang, J., Sun, J., \& Bondy, S. C. (2011). Paeonol increases levels of cortical cytochrome oxidase and vascular actin and improves behavior in a rat model of Alzheimer's disease. Brain Research, 1388, 141-7. [DOI:10.1016/j. brainres.2011.02.064] [PMID] 
This Page Intentionally Left Blank 